Farm animal welfare - who writes the rules?

Occasional Publication No. 23 - British Society of Animal Science 1999

edited by A. J. F. Russel, C. A. Morgan, C. J. Savory, M. C. Appleby and T. L. J. Lawrence

\title{
Evaluation of the effects of the positive 'befriending' of sows and gilts ('pleasant' treatment) prior to parturition and in early lactation on sow behaviour, the process of parturition and piglet survival
}

\author{
P. R. English, S. A. Grant, O. McPherson and S. A. Edwards \\ Department of Agriculture, University of Aberdeen, MacRobert Building, 581 King Street, Aberdeen AB24 5UA
}

\section{Introduction}

There is evidence that pleasant handling of pigs relative to aversive handling has beneficial influences on reproduction, growth, animal physiology and indices of welfare (Hemsworth et al., 1986 and 1987). However, there are no objective data available on the effect of pleasant handling of sows prior to parturition on the progress and efficiency of the parturition process and on subsequent piglet survival. The response to pleasant, relative to aversive, handling of pigs has been shown to be an effect of reducing fear responses to humans and the alleviation of a chronic stress response which has been associated with aversive handling. In indoor pig production, normal pre-farrowing practice involves the transfer of sows from pregnancy to farrowing accommodation around 4 to 7 days before the calculated farrowing date. The most common type of indoor farrowing facility is a pen with crate system, the crate being deployed to accommodate and restrain the sow so as to reduce the risk of piglets being overlain. However, the confinement within the crate prior to parturition may have negative influences on the sow in some situations. Both Cronin et al. (1991) and Lawrence et al. (1993) have demonstrated higher corticosteroid levels, which are indicative of both acute and chronic stress, in crated sows without bedding relative to loose housed sows with bedding prior to parturition. Prefarrowing stress can affect the efficiency of parturition through hyperactivity of the adrenal cortex, increased adrenalin secretion and depressed oxytocin secretion from the anterior pituitary gland. Prolonged parturition, an increased incidence of intrapartum stillbirths and a higher incidence of anoxia in live-born piglets (especially the later births) is likely to result.

There is evidence from studies on sows in an aversive housing system in pregnancy, viz. individual confinement in tether stalls, that pleasant handling can significantly reduce chronic stress levels relative to aversive and even minimal (neutral) handling treatments (Pedersen et al., 1999). Thus, it is possible that pleasant handling applied to sows prior to parturition and continued during and for a period after parturition might help to reduce stress levels at this time with consequent benefits to the sow, the efficiency of parturition and piglet survival.

Moreover, it is possible that any stress in the sow around parturition is not induced solely by confinement within the crate. The process of removing the sow from her pregnancy accommodation, isolating her from her penmates and guiding her along unfamiliar passage ways and eventually into a farrowing crate could induce acute stress, and more especially (a) in the case of gilts due to farrow for the first time and (b) where a high proportion of negative behaviours may be deployed by some stockpeople in 'persuading' the sow or gilt to move from pregnancy to farrowing accommodation. Thus the influence of pleasant handling applied for a short period each day from late pregnancy to just after farrowing was evaluated both in gilts farrowing for the first time and in older sows relative to a 'minimal' or 'neutral' treatment as the control.

\section{Material and methods}

The study took place in a 500 sow commercial herd with hybrid sows. Parity 1 pregnant gilts were loose housed in pen groups of six to eight and were given food on the floor once daily. Sows in their second and later pregnancy were housed in individual stalls and were also given food once daily. In general, sows on this farm were handled carefully and gently by the stockpeople. However, the handling of gilts and sows during pregnancy could be described as 'minimal' since the daily food was dispensed from outwith the pen or stall and there had been no direct tactile contact with the sows since they were served. Pleasant handling of the sows was applied in the pregnancy accommodation from 7 days before transfer to the farrowing quarters and this continued up to farrowing and for 2 days subsequently. The pleasant handling or 'befriending' took the form of 
close contact, talking to the sows and hand contact to stroke or to rub them. Approximately $1 \mathrm{~min}$ was spent in such befriending per animal per day. In the case of the pregnant gilts, handling took the form of entering the pen and 'befriending' each of the gilts in turn. Thus the interaction with the gilts was of a tactile, visual, auditory and olfactory nature, and 6 to $8 \mathrm{~min}$ was spent in each pen daily according to the number of gilts accommodated in it. It was more difficult to achieve such close contact with the sows in the stalls. The experimenter knelt down in front of each stall for $1 \mathrm{~min}$, making gentle hand contact with each sow as it approached the extended hand while talking quietly at the same time.

A block of adjacent sows in the stalls which were due to farrow at the same time was randomly allocated to either the pleasant $(\mathrm{P})$ or control $(\mathrm{C})$ treatment, while pens of gilts were also randomly allocated to treatment. Individual farrowing houses containing 12 or 16 pens contained only C or only P treatment sows. Sows were considered to be receptive to the befriending of the experimenter when they approached the stationary observer in the pen (gilts) or at the front of the stall (multiparous sows) and also on the basis of their vocalizations. In contrast, sows were considered to be nervous of the experimenter when they did not approach or retreated from the stationary observer and on the basis of their vocalizations. In the process of transferring sows from pregnancy to farrowing accommodation, each was handled in a standard manner by the experimenter. Each sow was scored subjectively for 'ease of handling' (easy, moderate, difficult), while an approach behaviour test to assess level of fear was carried out on each sow in a standard test arena en route between pregnancy and farrowing accommodation.

Monitoring and supervision of farrowings were carried out by farm staff according to their normal routine. The experimenter was opportunistic in monitoring the progress of farrowings, recording the timing of as many piglet births as possible so as to determine birth intervals. There were $33 \mathrm{C}$ sows and gilts and 27 subjected to the $P$ treatment, with the great majority being in parities 1 and 3 .

Frequencies were compared using the Fisher exact probablity test. Other parameters were compared by analysis of variance using the sow as the experimental unit in all cases.

\section{Results}

When befriending of the gilts and sows was started 7 days before entry into the farrowing rooms, a very high proportion of the gilts $(93 \cdot 1 \%)$ were classified as

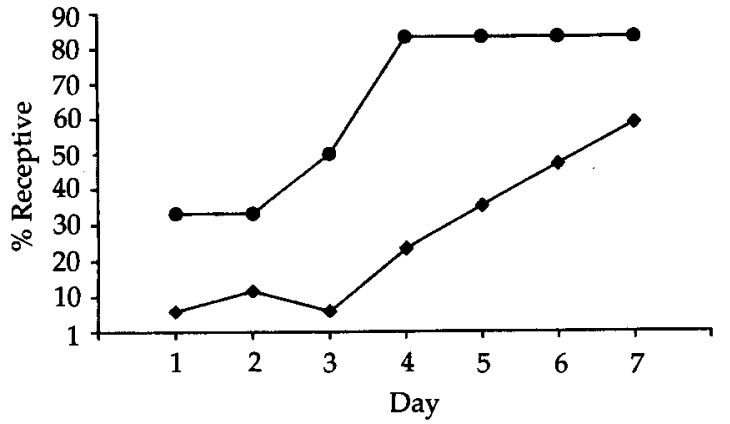

Figure 1 Trends in the percentage of gilts and parity 3 sows tested which were receptive to daily befriending by the stockperson (in pregnancy accommodation): - - parity 3 (no. $=10) ; \longrightarrow$ parity 1 (no. $=17$ ).

Table 1 Ease of handling and approach behaviour scorest

\begin{tabular}{lccc}
\hline \hline & Control & Pleasant & $\chi^{2}$ \\
\hline Easy to move (\%) & & & \\
Parity 1 & 50 & 69 & $0 \cdot 05$ \\
Parity 3 & 92 & 100 & $0 \cdot 25$ \\
Approach time (s) & & & s.e.d. \\
Parity 1 & 54 & 46 & $33 \cdot 5$ \\
Parity 3 & 65 & 32 & 36.5 \\
\hline \hline
\end{tabular}

$\dagger$ No differences were statistically significant $(P>0.05)$.

Table 2 Proportion of sows making savaging attempts during parturition

\begin{tabular}{lccc}
\hline \hline & Control & Pleasant & $\chi^{2}$ \\
\hline Parity 1 & $22 \cdot 2$ & 0 & 4.55 \\
Parity 3 & 13.0 & 0 & 0.87 \\
All parities & 15.2 & $7 \cdot 4$ & 0.28 \\
\hline \hline
\end{tabular}

+ No differences were statistically significant $(P>0.05)$.

being nervous/very nervous at being handled in the 'befriending' process (see Figure 1). However, fairly quickly both gilts and sows became receptive to the befriending process. Thus on the first day, only $5.9 \%$ of the gilts were receptive to the befriending process but by day $7,58.9 \%$ of these gilts were receptive. The respective figures for parity 3 sows were $33.3 \%$ on day 1 and $83.3 \%$ on day 7 . The proportion of gilts and sows which became receptive to daily befriending continued to increase in the farrowing accommodation prior to parturition.

In the transfer from pregnancy to farrowing accommodation, $\mathbf{P}$ sows achieved higher 'ease of handling' and 'approach behaviour' scores in a test arena but none of these differences approached statistical significance (see Table 1). During 
Table 3 Mean interval ( $\mathrm{min}$ ) between births of successive piglets

\begin{tabular}{lccc}
\hline \hline & Control & Pleasant & s.e.d. \\
\hline Parity 1 & 20.9 & $13.9 * *$ & $2 \cdot 3$ \\
Parity 3 & 21.7 & $17 \cdot 9$ & 6.0 \\
All parities & 20.7 & $15 \cdot 6$ & $3 \cdot 4$ \\
\hline \hline
\end{tabular}

Table 4 The incidence of stillbirths and live-birth lossest

\begin{tabular}{|c|c|c|c|}
\hline & Control & Pleasant & s.e.d. \\
\hline Stillbirths per litter & 0.76 & 0.52 & 0.21 \\
\hline Live births per litter & $11 \cdot 18$ & $10 \cdot 74$ & 0.69 \\
\hline Livebirth losses (\%) & & & $\chi^{2}$ \\
\hline Before fostering & $1 \cdot 1$ & 0.7 & \\
\hline From fostering up to 14 days & 3.9 & $2 \cdot 6$ & $1 \cdot 12$ \\
\hline III thriving pigs fostered off & $11 \cdot 2$ & $11 \cdot 3$ & 0.66 \\
\hline
\end{tabular}

+ No differences were statistically significant $(P>0.05)$.

parturition, the proportion of sows which made savaging attempts on newborn piglets was consistently lower in the $\mathbf{P}$ group (see Table 2).

On the basis of 196 and 143 birth intervals observed in the $C$ and $P$ groups respectively, birth intervals were consistently lower in $P$ (see Table 3 ).

The difference between $C$ and $P$ in parity 1 was highly significant. The incidence of stillbirths and live-birth losses were lower in $\mathrm{P}$ but these differences were not significant (see Table 4).

Fostering practice was efficient on this farm, and at the first signs of piglets failing to compete within a litter, with body condition loss, such piglets were fostered on to newly farrowed non-experimental sows with spare rearing capacity. The great majority of these fostered piglets survived.

Thus, there were consistent indications in the results that the $P$ treatment had desirable influences in reducing fear of humans, on the ease of handling and on the physiology of the sow, as indicated by the reduced intervals between successive piglet births. Mortality in terms of stillbirths and live-birth losses was also marginally but not significantly lower on $P$.

\section{Discussion}

There were indications from this study that the pleasant or 'befriending' treatment applied reduced fear of humans, improved ease of handling, reduced birth intervals and the incidence of savaging during parturition, while there were marginal reductions in the incidence of both stillbirths and live-birth losses. Only some of these differences were significant but all the trends were entirely consistent in terms of indicating positive responses from the pleasant treatment.

Although the pigs were, in general, well handled on this farm, it is likely that the pregnant gilts and sows involved in the study could be classified up to the time the pleasant treatment was applied as being on a 'minimal' handling treatment as described by Hemsworth et al. (1986). They were given food once daily from outside the pen or stall but had not been handled (tactile contact with humans) since they were served at the start of their pregnancy. Since it is the nature and number of the interactions (and particularly those of a tactile nature) which determine the quality of the human-animal relationship for the animal (Hemsworth and Coleman, 1998), it could be argued that the sows and gilts in this study had been subjected to no positive or negative tactile interactions with humans for approximately 100 days since mating, and therefore their human treatment up to this time was neither pleasant nor aversive, but was 'minimal' . Barnett et al. (1994) with poultry and Gonyou et al. (1986) and Grandin et al. (1987) with pigs, found that a high level of fear can result from reduced human contact. While such contact can be of a visual, auditory, olfactory, gustatory and tactile nature, it appears that the fear and stress responses of the animal to the human is particularly affected by the tactile interactions (Hemsworth et al., 1981; Gonyou et al., 1986; Pearce et al., 1989).

That the sows in the present study appeared to be fearful of humans at the start of treatment is indicated by their very low initial response to befriending. Thus, $94.3 \%$ of the gilts and $66.7 \%$ of the sows were classified as being very nervous in response to the initial attempts at befriending by the experimenter. In other words, a high proportion of these gilts and sows were demonstrating a fear response. However, the proportion of sows responding to the daily befriending increased quickly, indicating a rapid reduction in fear responses. The rapid response to befriending with a daily time input of only $1 \mathrm{~min}$ per animal is surprising at first sight, although in the studies of Hemsworth et al. (1981), Gonyou et al. (1986) and Pearce et al. (1989), pleasant handling treatments applied for only 15 to $30 \mathrm{~s}$ per animal daily were effective in reducing fear responses and alleviating chronic stress.

In the current study, while sows received direct befriending for only $1 \mathrm{~min}$ daily, it is likely that each gilt in the pen groups of six to eight was, in fact, 
inadvertently receiving this treatment for 6 to $8 \mathrm{~min} /$ day. With the experimenter in the pen of gilts handling and stroking each one gently in turn and speaking quietly at the same time, all the gilts in the pen had comprehensive visual, auditory and olfactory and often also tactile contact with the experimenter. The fact that the responses to pleasant handling obtained from the gilts appeared to be slightly greater than those obtained from older sows may have been due to this factor of the duration of effective 'befriending' per day, or it may have been that the gilts, with less experience of previous handling by humans, had higher fear and chronic stress levels at the start of the treatment. This may have accentuated the differences between the pleasant and control treatments in gilts relative to the differences found in the sows.

In the process of moving the gilts and sows from the pregnancy pens to the farrowing pens, the befriended animals had higher 'ease of handling' and approach behaviour scores which were consistent, but were not significantly different from the controls. The isolation of individual gilts from their pregnancy pen group, herding along strange passage ways and eventually into the confinement of a farrowing crate, is likely to be a fear-provoking experience for many animals which is likely to result in acute and chronic stress responses. After transfer to the farrowing house, if gilts have not developed trust in their human carers as a result of reduced fear levels, then each time the stockperson enters the house and the farrowing pen and handles the gilt, e.g. to test for the present of colostrum and the imminence of farrowing, this is likely to induce acute stress responses until such regular pleasant daily handling results in reduction of fear levels and stress responses. In the absence of regular pleasant handling, the isolation and restraint within the farrowing crate, combined with pain and fear induced by the onset of parturition and contractions, are factors which are likely to increase acute and chronic stress levels. It is possible that the higher incidence of savaging of piglets, the significantly higher intervals between births and the marginally higher stillbirth rate in the control treatment are the outcomes of such stress responses.

Thus, the indications are that the parity 1 gilts on the pleasant treatment were less fearful and easier to handle than controls as they were guided from their original pregnancy pen to their farrowing accommodation. Kiley-Worthington (1990), in describing the handling of circus animals to reduce their fear of humans, has found that when a trusting animal-human relationship is developed, the animal will 'go places and do things with the handler that it would be otherwise frightened to do'.
Therefore, the parity 1 gilts on the pleasant treatment may have suffered less acute stress than the controls as they were being transferred from pregnancy to farrowing accommodation and less chronic stress when they were confined in the farrowing pen. The alternative interpretation is that the chronic stress associated with minimal human handling during pregnancy was alleviated more by the pleasant handling than on the control treatment in late pregnancy and during parturition.

A complication in interpreting the outcome of this study was that, while the experimenter applied the pleasant handling treatment and was involved in transferring all sows from pregnancy to farrowing accommodation, farm staff assisted with this transfer and were also involved in the normal commercial checks on testing for the presence of milk and imminence of parturition, and for monitoring the progress of farrowing as their other duties allowed. Thus, the independent influences of the experimenter and the farm staff cannot be determined.

It is possible that the gilts subjected to pleasant handling demonstrated stimulus generalization (Hemsworth et al., 1993). This is where the animal generalizes about humans in general from their experience with the most influential human in their lives or the most recent period in their life. So that, if they had been handled pleasantly by their main human contact and have had low fear and stress responses to this contact and handling, then they will show similar responses to other humans. However, there is some evidence that if animals have been subjected to pleasant treatment by one human contact and to aversive treatment of the same degree by another human, then fear responses to humans in general are likely to be more influenced by their responses to the more aversive of the two handlers (Hemsworth et al., 1994; de Passille et al., 1996).

However, since the gilts and sows on this farm appeared to be subjected to 'minimal' rather than aversive treatment during pregnancy, it is possible that the responses of the pleasant treatment sows to the farm staff in late pregnancy and during parturition were similar to those exhibited in response to the experimenter. Thus, in the case of the pleasant treatment, the presence of, and tactile contact with, any of the farm staff and the experimenter in the farrowing pen before and during farrowing might have been effective in helping to alleviate a chronic stress response relative to the sows on the control treatment.

While the outcome of this study tends to pose more questions than it answers, the results were generally 
promising, and there is a need to extend the studies to larger numbers of sows per treatment and to replicate the studies over a range of commercial conditions involving different housing systems, personnel and physiological status of sows, especially in terms of chronic stress levels.

\section{References}

Barnett, J. L., Hemsworth, P. H., Hennessy, D. P., McCallum, T. M. and Newman, E. A. 1994. The effects of modifying the amount of human contact on the behavioural, physiological and production responses of laying hens. Applied Animal Behaviour Science 41: 87-100.

Cronin, G. M., Barnett, J. L., Hodge, F. M., Smith, J. A. and McCallum, T. H. 1991. The welfare of pigs in two farrowing/lactation environments: cortisol responses of sows. Applied Animal Behaviour Science 32: 117-127.

Gonyou, H. W., Hemsworth, P. H. and Barnett, J. L. 1986. Effects of frequent interactions with humans on growing pigs. Applied Animal Behaviour Science 16: 269-278.

Grandin, T., Curtis, S. E. and Taylor, I. A. 1987. Toys, mingling and driving reduce excitability in pigs. Journal Animal Science 65: (Suppl. 1), 230 (abstr.).

Hemsworth, P. H., Barnett, J. L. and Hansen, C. 1981. The influence of handling by humans on the behaviour, growth and corticosteriods in the juvenile female pig. Hormones and Behaviour 15: 395-403.

Hemsworth, P. H., Barnett, J. L. and Hansen, C. 1986. The influence of handling by humans on the behaviour, reproduction and corticosteriods of male and female pigs. Applied Animal Behaviour Science 15: 303-314.

Hemsworth, P. H., Barnett, J. L. and Hansen, C. 1987. The influence of inconsistent handling by humans on the behaviour, growth and corticosteriods of young pigs. Applied Animal Behaviour Science 17: 245-252.

Hemsworth, P. H., Barnett, J. L. and Coleman, G. J. 1993. The human-animal relationship in agriculture and its consequences for the animal. Animal Welfare 2: 33-51.

Hemsworth, P. H., Coleman, G. J., Cox, M. and Barnett, J. L. 1994. Stimulus generalisation: the inability of pigs to discriminate between humans on the basis of their previous handling experience. Applied Animal Behaviour Science 40: 129-142.

Hemsworth, P. H. and Coleman, G. J. 1998. Human-livestock interactions: the stockperson and the productivity and welfare of intensively farmed animals. CAB International, Wallingford.

Kiley-Worthington, M. 1990. Animals in circuses and zoos. Chiron's World, Little Eco-Farms Publishing, Basildon, Essex.

Lawrence, A. B., Petherick, J. C., McLean, K. A., Deans, L. A., Chirnside, J. and Vaughan, A. 1993. The effect of the environment on plasma cortisol in parturient sows. Animal Production 56: 476 (abstr.).

Passille, A. M. de, Rushen, J., Ladewig, J. and Petherick, C. 1996. Dairy calves' discrimination of people based on previous handling. Journal of Animal Science 74: 969-974.

Pearce, G. P., Paterson, A. M. and Pearce, A. N. 1989. The influence of pleasant and unpleasant handling and the provision of toys on the growth and behaviour of male pigs. Applied Animal Behaviour Science 23: 27-37.

Pedersen, V., Barnett, J. L., Hemsworth, P. H., Newman, E. A. and Schirmer, B. 1999. The effects of handling on behavioural and physiological responses to housing in tether-stalls in pregnant sows. Animal Welfare In press. 